\title{
Escarabeíneos copro-necrófagos (Coleoptera, Scarabaeidae, Scarabaeinae) de fragmentos de Mata Atlântica em Silveira Martins, Rio Grande do Sul, Brasil
}

\author{
Pedro G. da Silva ${ }^{1,2} \&$ Rocco A. Di Mare ${ }^{2}$ \\ 1. Programa de Pós-Graduação em Ecologia, Universidade Federal de Santa Catarina, Rua João Pio Duarte Silva, Córrego Grande, 88040-900, Florianópolis, SC, Brasil. \\ (pedrogiovanidasilva@yahoo.com.br) \\ 2. Programa de Pós-Graduação em Biodiversidade Animal, Universidade Federal de Santa Maria, Avenida Roraima, 1000, Camobi, 97105-900, Santa Maria, RS, Brasil. (ram13@terra.com.br)
}

\begin{abstract}
Copro-necrophagous beetles (Coleoptera: Scarabaeidae: Scarabaeinae) from Atlantic Forest fragments of Silveira Martins, Rio Grande do Sul, Brazil. The fauna of Scarabaeinae (Coleoptera: Scarabaeidae) was sampled using pitfall traps baited with human excrement and rotten fish in forest fragments in Silveira Martins, Rio Grande do Sul, Brazil, from November 2010 to January 2011. A total of 1,611 individuals were sampled, belonging to six tribes, 11 genera and 28 species. The most abundant species were Canthon latipes Blanchard, 1845 (49.9\%), C. chalybaeus Blanchard, 1845 (13.9\%), Deltochilum sculpturatum Felsche, 1907 (4.9\%), and Eurysternus caribaeus (Herbst, 1789) (4.3\%), which accounted for $73 \%$ of the total individuals captured. Traps baited with human excrement captured a higher number of species than those baited with rotten fish. There was no statistically significant difference between the kinds of baits used in relation to the abundance of Scarabaeinae. Most of Scarabaeinae species was represented by generalist feeding habits and tunneler behavior. The community sampled follows the general patterns of trophic and behavioral structure of Scarabaeinae found throughout the Neotropical region.
\end{abstract}

KEYWORDS. Food preference, coprophagous beetle, necrophagous beetle, south of Brazil.

RESUMO. A fauna de Scarabaeinae (Coleoptera: Scarabaeidae) foi amostrada através de armadilhas de queda iscadas com excremento humano e peixe apodrecido em fragmentos florestais de Silveira Martins, Rio Grande do Sul, Brasil, de novembro de 2010 a janeiro de 2011. Foi coletado um total de 1.611 indivíduos, pertencentes a seis tribos, 11 gêneros e 28 espécies. As espécies mais abundantes foram Canthon latipes Blanchard, 1845 (49,9\%), C. chalybaeus Blanchard, 1845 (13,9\%), Deltochilum sculpturatum Felsche, 1907 (4,9\%) e Eurysternus caribaeus (Herbst, 1789) $(4,3 \%)$, que juntas representaram $73 \%$ do total de indivíduos capturados. As armadilhas iscadas com excremento humano capturaram maior número de espécies do que as iscadas com peixe apodrecido. Não houve diferença estatística significativa entre os tipos de iscas utilizados em relação à abundância de Scarabaeinae. A maior parte da comunidade de Scarabaeinae capturada foi representada por espécies de hábito alimentar generalista e comportamento escavador. A comunidade amostrada segue os padrões gerais de estrutura trófica e comportamental de Scarabaeinae encontrados por toda a região Neotropical.

PALAVRAS-CHAVE. Preferência alimentar, besouro coprófago, besouro necrófago, sul do Brasil.

A subfamília Scarabaeinae (Coleoptera: Scarabaeidae) agrupa besouros detritívoros que atuam nos processos biológicos dos ecossistemas terrestres, contribuindo com a decomposição de matéria orgânica e a reciclagem de nutrientes (Nichols et al., 2008). Existem aproximadamente 7.000 espécies descritas de escarabeíneos em todo o mundo (SchoolmeEsters et al., 2010), sendo muito diversos em florestas e savanas tropicais (Halffter \& MatTHEws, 1966; HANSKi \& Cambefort, 1991; Simmons \& Ridsdill-Smith, 2011).

Os escarabeíneos utilizam principalmente excrementos de mamíferos (coprofagia), carcaças de animais (necrofagia) e matéria vegetal em decomposição (saprofagia) na nidificação e alimentação de larvas e adultos (Halffter \& Matthews, 1966; Hanski \& CAmbefort, 1991; Simmons \& Ridsdill-Smith, 2011). Podem enterrar porções do alimento em galerias construídas logo abaixo do recurso (escavadores), rolálas por vários metros até serem enterradas (roladores), ou nidificar no interior do alimento (residentes) (Halffter \& Matthews, 1966; Halffter \& Edmonds, 1982; Simmons \& RidSDill-Smith, 2011).

Em florestas e savanas tropicais, os escarabeíneos têm sido utilizados como um eficiente grupo bioindicador, uma vez que suas assembleias podem responder negativamente a alterações naturais ou antrópicas (HALFFTER \& FAVILA, 1993; DAVIS et al., 2004; LaRSEN \& Forsyth, 2005; Nichols et al., 2007; GARDNER et al., 2008; Neves et al., 2010; Filgueiras et al., 2011). Tais alterações podem resultar em mudanças na riqueza, composição, abundância e diversidade de Scarabaeinae (Klein, 1989; Halffter \& Arellano, 2002; Escobar et al., 2007).

Em geral, o conhecimento sobre a maioria dos grupos de invertebrados no Rio Grande do Sul ainda é insuficiente, sendo os lepidópteros e himenópteros os mais bem conhecidos (Bencke, 2009). Em Coleoptera, somente Cerambycidae e Chrysomelidae têm sido bem estudados (Moura, 2003). Já trabalhos sobre Scarabaeinae ainda são escassos, mas destacam-se os de SiLva et al. (2008, 2009), Audino et al. (2011) e SiLVA (2011). Como na maioria dos estados brasileiros, o Rio Grande do Sul carece de levantamentos, necessitando de mais amostragens para caracterizar melhor a fauna e distribuição de Scarabaeinae (VAZ-DE-Mello, 2000). Tais estudos contribuirão para o conhecimento sobre a real distribuição das espécies nos biomas e ecossistemas do território brasileiro, assim como trarão novas informações sobre a biologia das espécies de escarabeíneos. 
Dessa forma, o objetivo deste estudo foi ampliar o conhecimento sobre as espécies de Scarabaeinae (riqueza e abundância) e comparar a atratividade das iscas de excremento humano e peixe em decomposição em fragmentos de Mata Atlântica no Rio Grande do Sul, Brasil.

\section{MATERIAL E MÉTODOS}

O estudo foi desenvolvido em três áreas florestais em meio a uma paisagem fragmentada de Mata Atlântica na localidade de Val Feltrina no município de Silveira

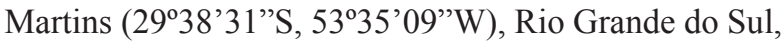
Brasil. Este município localiza-se na encosta do rebordo do Planalto Meridional Brasileiro, na área de transição com a Depressão Central do estado, dentro do bioma Mata Atlântica, possuindo uma área aproximada de 119 $\mathrm{km}^{2}$ (IBGE, 2011). O município possui clima subtropical do tipo Cfa conforme a classificação de Köppen-Geiger (Pell et al., 2007). A altitude varia entre 100 e $480 \mathrm{~m}$, e a temperatura média anual é de aproximadamente $18^{\circ} \mathrm{C}$, com temperaturas mínimas próximas a $0^{\circ} \mathrm{C}$ e máximas próximas de $40^{\circ} \mathrm{C}$. A precipitação média anual varia entre $1.500 \mathrm{e} 1.700 \mathrm{~mm}$ aproximadamente. A localidade de Val Feltrina constitui-se de um vale entre conjuntos de montanhas que possuem altitudes entre 300 e 400 $\mathrm{m}$, e entre 100 e $180 \mathrm{~m}$ na parte mais baixa do vale. A vegetação pertence à floresta estacional decidual, no domínio do bioma Mata Atlântica. A região é composta de áreas agrícolas e pecuárias na parte baixa do vale, cercada pela vegetação florestal presente na encosta dos morros. Neste contexto foram escolhidas três áreas amostrais de formas irregulares ligadas a uma matriz florestal, representadas pelo mesmo tipo e estado de conservação da vegetação.

Os escarabeíneos foram capturados através de armadilhas de queda iscadas, pois estas correspondem ao método de coleta mais eficiente para a captura da maior parte desta fauna (LoBo et al., 1988; HalfFTER \& FAVILA, 1993). As armadilhas foram compostas de um recipiente plástico de $1.000 \mathrm{ml} \mathrm{com} 13 \mathrm{~cm}$ de diâmetro e $10 \mathrm{~cm}$ de altura, que foram enterradas de modo que a borda ficasse ao nível do solo, permitindo a queda dos insetos (LoBo et al., 1988). Um pote plástico de menores dimensões $(50 \mathrm{ml})$ foi colocado sobre o maior para acomodação das iscas (aproximadamente $25 \mathrm{~g}$ ). Foram utilizados pratos plásticos amparados por estacas de madeira para a proteção da armadilha contra a chuva. Dentro de cada recipiente coletor foi utilizada solução de água, formalina (10\%) e detergente (neutro) para capturar e preservar os espécimes. Para atrair as espécies coprófagas e necrófagas de Scarabaeinae foram utilizadas fezes humanas e peixe em decomposição (apodrecida em potes plásticos por três dias em temperatura ambiente antes de cada amostragem).

A amostragem foi realizada entre 15 de novembro de 2010 (instalação) e 30 de janeiro de 2011, em visitas quinzenais, totalizando cinco coletas. As armadilhas permaneceram em campo durante todo o período de estudo. O período escolhido para a realização das amostragens corresponde à época de maior abundância (primavera-verão) de Scarabaeinae em regiões de clima subtropical (HALFFTER \& MatThews, 1966).

Em cada área amostral foram distribuídas oito armadilhas em pares, onde cada par continha os dois tipos de iscas utilizados. As armadilhas foram distanciadas dois metros entre si, e os conjuntos ficaram distantes 30 $\mathrm{m}$ entre si.

Os besouros capturados foram levados ao Laboratório de Biologia Evolutiva da Universidade Federal de Santa Maria (UFSM), onde foram triados, montados, contados e identificados. A identificação foi realizada com auxílio de chave dicotômica para gêneros de Scarabaeinae (VAZ-DE-Mello et al., 2011), comparação com espécimes depositados no laboratório e alguns espécimes foram enviados para o especialista do grupo Dr. Fernando Vaz de Mello na Universidade Federal de Mato Grosso (UFMT), Brasil. Os insetos estão depositados nas coleções do Laboratório de Biologia Evolutiva da UFSM e coleção pessoal Fernando Z. Vaz de Mello da UFMT.

A proximidade das armadilhas iscadas com diferentes recursos permitiu a escolha pelos escarabeíneos (Almeida \& LouzAdA, 2009). Assim, os indivíduos foram classificados como: coprófagos, quando $80 \%$ dos indivíduos foram capturados em armadilhas com fezes; necrófagos, quando $80 \%$ dos indivíduos foram capturados em armadilhas com peixe apodrecido; e generalistas, as espécies que não se enquadraram nos grupos anteriores (Halffter \& Arellano, 2002; Almeida \& Louzada, 2009). Para o último grupo, a preferência pela coprofagia ou necrofagia foi destacada quando a proporção de indivíduos fosse igual ou superior a $60 \%$ e menor do que $80 \%$ em um dos tipos de armadilhas iscadas. Espécies com um (singleton) ou dois indivíduos (doubleton) não foram classificadas nas categorias alimentares por não haver número de indivíduos suficiente para esta inferência (Almeida \& Louzada, 2009).

As espécies foram classificadas em guildas comportamentais, ou seja, o modo como utilizam o recurso para a alimentação e nidificação, sendo agrupadas em escavadoras, roladoras e residentes, conforme a literatura (HALFFTER \& EDMONDS, 1982; CAMBEFORT \& Hanski, 1991; Doube, 1991; Gill, 1991; Koller et al., 2007; Silva et al., 2008, 2009; Silva, 2011; Simmons \& RidSDill-SMith, 2011).

A distribuição da abundância das espécies de Scarabaeinae foi comparada graficamente entre os tipos de armadilhas iscadas com diferentes recursos através de ranques para comparar padrões de distribuição da abundância e equitabilidade entre as espécies (FEINSINGER, 2001; VERDÚ et al., 2007), onde a abundância relativa de cada espécie foi transformada em logaritmo de base 10 $(\mathrm{N}+1)$ e disposta do maior para o menor valor para cada habitat (VERDÚ et al., 2007). 
O método de rarefação (Hulbert, 1971), o qual consiste em calcular o número esperado de espécies em cada amostra para um tamanho de amostra padrão, foi utilizado com o objetivo de padronizar o número de indivíduos e comparar a riqueza encontrada nos dois tipos de iscas utilizados, sendo efetuado no programa PAST 2.02 (Hammer et al., 2001). O teste de Kruskal-Wallis foi utilizado para verificar possíveis diferenças na abundância de Scarabaeinae pelos tipos de iscas utilizados, sendo conduzido no programa BioEstat 5.0 (Ayres et al., 2007).

A fim de obter estimativas da riqueza a ser encontrada em cada tipo de armadilha iscada e no total (paisagem), foram calculados os estimadores de riqueza Bootstrap, Chao 1 e 2, e Jackknife 1 e 2, com 500 randomizações com reposição de amostras, no programa EstimateS 7.0 (Colwell, 2004), para se obter um maior número de estimativas devido à variabilidade dos parâmetros levados em maior consideração pelos diferentes estimadores (Cowell \& Coddington, 1994).

\section{RESULTADOS}

Foi coletado um total de 1.611 indivíduos pertencentes a seis tribos, 11 gêneros e 28 espécies (Tab. I). As espécies mais abundantes foram Canthon latipes Blanchard, 1845 (49,9\%), C. chalybaeus Blanchard, 1845 (13,9\%), Deltochilum sculpturatum Felsche, 1907 (4,9\%) e Eurysternus caribaeus (Herbst, 1789) $(4,3 \%)$, que representaram $73 \%$ do total de indivíduos capturados. Deltochilini foi a tribo mais representativa no estudo, abrigando 12 espécies de dois gêneros, e $82,56 \%$ do total de indivíduos capturados.

Tab. I. Tribos e espécies de Scarabaeinae capturadas com armadilhas de queda iscadas com excremento humano e peixe apodrecido em três áreas florestais em Silveira Martins, Rio Grande do Sul, Brasil, entre novembro de 2010 e janeiro de 2011 (TPA, total em armadilhas iscadas com peixe apodrecido; TEX, total em excremento humano; TG, total geral; \%, porcentagem em relação ao total geral; PA, peixe apodrecido; EX, excremento humano; S, riqueza; N, abundância).

\begin{tabular}{|c|c|c|c|c|c|c|c|c|c|c|}
\hline \multirow{3}{*}{ TRIBO/espécie } & \multicolumn{6}{|c|}{ Fragmentos } & \multirow{3}{*}{ TPA } & \multirow{3}{*}{ TEX } & \multirow{3}{*}{ TG } & \multirow{3}{*}{$\%$} \\
\hline & \multicolumn{2}{|c|}{1} & \multicolumn{2}{|c|}{2} & \multicolumn{2}{|c|}{3} & & & & \\
\hline & PA & $\mathrm{EX}$ & PA & EX & PA & EX & & & & \\
\hline \multicolumn{11}{|l|}{ ATEUCHINI $(\mathrm{S}=2 ; \mathrm{N}=2)$} \\
\hline Ateuchus aff. carbonarius (Harold, 1868) & 0 & 0 & 0 & 0 & 1 & 0 & 1 & 0 & 1 & 0,06 \\
\hline Uroxys aff. dilaticollis Blanchard, 1845 & 0 & 0 & 0 & 0 & 0 & 1 & 0 & 1 & 1 & 0,06 \\
\hline \multicolumn{11}{|l|}{ COPRINI $(\mathrm{S}=8 ; \mathrm{N}=80)$} \\
\hline Canthidium aff. dispar Harold, 1867 & 1 & 3 & 0 & 0 & 0 & 1 & 1 & 4 & 5 & 0,31 \\
\hline Canthidium aff. trinodosum (Boheman, 1858) & 1 & 6 & 2 & 4 & 2 & 3 & 5 & 13 & 18 & 1,12 \\
\hline Canthidium sp. & 4 & 21 & 1 & 6 & 5 & 10 & 10 & 37 & 47 & 2,92 \\
\hline Dichotomius aff. acuticornis (Luederwaldt, 1930) & 0 & 0 & 0 & 0 & 1 & 1 & 1 & 1 & 2 & 0,12 \\
\hline Dichotomius assifer (Eschscholtz, 1822) & 0 & 0 & 0 & 0 & 1 & 1 & 1 & 1 & 2 & 0,12 \\
\hline Dichotomius nisus (Olivier, 1789) & 0 & 1 & 0 & 0 & 0 & 1 & 0 & 2 & 2 & 0,12 \\
\hline Ontherus azteca Harold, 1869 & 0 & 1 & 0 & 0 & 0 & 1 & 0 & 2 & 2 & 0,12 \\
\hline Ontherus sulcator (Fabricius, 1775) & 0 & 0 & 0 & 0 & 0 & 2 & 0 & 2 & 2 & 0,12 \\
\hline \multicolumn{11}{|l|}{ DELTOCHILINI $(\mathrm{S}=12 ; \mathrm{N}=1.330)$} \\
\hline Canthon amabilis Balthasar, 1939 & 5 & 5 & 3 & 2 & 14 & 20 & 22 & 27 & 49 & 3,04 \\
\hline Canthon chalybaeus Blanchard, 1845 & 41 & 53 & 4 & 4 & 72 & 50 & 117 & 107 & 224 & 13,9 \\
\hline Canthon latipes Blanchard, 1845 & 48 & 106 & 12 & 39 & 242 & 357 & 302 & 502 & 804 & 49,9 \\
\hline Canthon lividus Blanchard, 1845 & 4 & 7 & 0 & 1 & 24 & 8 & 28 & 16 & 44 & 2,73 \\
\hline Canthon aff. luctuosus Harold, 1868 & 12 & 13 & 6 & 1 & 11 & 4 & 29 & 18 & 47 & 2,92 \\
\hline Canthon oliverioi Pereira \& Martínez, 1956 & 0 & 1 & 0 & 0 & 0 & 2 & 0 & 3 & 3 & 0,19 \\
\hline Canthon quinquemaculatus (Castelnau, 1840) & 2 & 0 & 0 & 0 & 0 & 0 & 2 & 0 & 2 & 0,12 \\
\hline Canthon laminatus Balthasar, 1939 & 1 & 0 & 0 & 1 & 0 & 2 & 1 & 3 & 4 & 0,25 \\
\hline Deltochilum brasiliense (Castelnau, 1840) & 1 & 1 & 1 & 3 & 14 & 24 & 16 & 28 & 44 & 2,73 \\
\hline Deltochilum morbillosum Burmeister, 1848 & 0 & 0 & 0 & 0 & 4 & 0 & 4 & 0 & 4 & 0,25 \\
\hline Deltochilum rubripenne (Gory, 1831) & 0 & 1 & 0 & 0 & 15 & 9 & 15 & 10 & 25 & 1,55 \\
\hline Deltochilum sculpturatum Felsche, 1907 & 18 & 12 & 5 & 1 & 23 & 21 & 46 & 34 & 80 & 4,97 \\
\hline \multicolumn{11}{|l|}{ ONITICELLINI $(\mathrm{S}=2 ; \mathrm{N}=98)$} \\
\hline Eurysternus caribaeus (Herbst, 1789) & 3 & 18 & 0 & 7 & 7 & 35 & 10 & 60 & 70 & 4,35 \\
\hline Eurysternus parallelus Castelnau, 1840 & 2 & 4 & 0 & 0 & 0 & 22 & 2 & 26 & 28 & 1,74 \\
\hline \multicolumn{11}{|l|}{ ONTHOPHAGINI $(\mathrm{S}=2 ; \mathrm{N}=36)$} \\
\hline Onthophagus aff. hirculus Mannerheim, 1829 & 0 & 0 & 0 & 1 & 0 & 1 & 0 & 2 & 2 & 0,12 \\
\hline Onthophagus aff. tristis Harold, 1873 & 0 & 5 & 0 & 2 & 4 & 23 & 4 & 30 & 34 & 2,11 \\
\hline \multicolumn{11}{|l|}{ PHANAEINI $(\mathrm{S}=2 ; \mathrm{N}=65)$} \\
\hline Coprophanaeus saphirinus (Sturm, 1826) & 10 & 9 & 2 & 2 & 29 & 11 & 41 & 22 & 63 & 3,91 \\
\hline Phanaeus splendidulus (Fabricius, 1781) & 0 & 0 & 0 & 0 & 0 & 2 & 0 & 2 & 2 & 0,12 \\
\hline Número de indivíduos & 153 & 267 & 36 & 74 & 469 & 612 & 658 & 953 & 1611 & 100 \\
\hline Número de espécies & 15 & 18 & 9 & 14 & 17 & 25 & 21 & 25 & 28 & \\
\hline
\end{tabular}


Nas armadilhas iscadas com excremento humano, as espécies mais abundantes foram $C$. latipes $(52,7 \%), C$. chalybaeus (11,2\%), E. caribaeus (6,3\%) e Canthidium sp. $(3,9 \%)$, que responderam por $74,1 \%$ do total de indivíduos capturados com este tipo de isca. Nas armadilhas iscadas com peixe apodrecido, C. latipes $(45,9 \%), C$. chalybaeus $(17,8 \%)$, D. sculpturatum (7\%) e Coprophanaeus saphirinus (Sturm, 1826) (6,2\%) foram as espécies mais abundantes, e representaram $76,9 \%$ do total de espécimes coletados com este tipo de isca.

As espécies Ateuchus aff. carbonarius (Harold, 1868) (singleton), Canthon quinquemaculatus (Castelnau, 1840) (doubleton) e Deltochilum morbillosum Burmeister, 1848, ocorreram somente em armadilhas iscadas com peixe apodrecido. Canthon oliverioi Pereira \& Martínez, 1956, Dichotomius nisus (Olivier, 1789) (doubleton), Ontherus azteca Harold, 1869 (doubleton), Ontherus sulcator (Fabricius, 1775) (doubleton), Onthophagus aff. hirculus Mannerheim, 1829 (doubleton), Phanaeus splendidulus (Fabricius, 1781) (doubleton) e Uroxys aff. dilaticollis Blanchard, 1845 (singleton), foram capturadas somente em armadilhas iscadas com excremento humano.
A abundância das espécies de Scarabaeinae nos dois tipos de iscas utilizados apresentou distribuição semelhante (Fig. 1). Dessa forma, parece não ter havido diferenças na equitabilidade entre os tipos de iscas utilizadas.

Conforme a classificação para as guildas tróficas foram capturadas 12 espécies (42,9\%) generalistas, quatro $(14,3 \%)$ coprófagas e somente uma $(3,6 \%)$ necrófaga (Tab. II). Onze espécies (39,3\%) não puderam ser enquadradas em nenhuma categoria de hábito alimentar, pois não tiveram número de indivíduos suficientes, sendo oito delas $(28,6 \%)$ doubletons e três delas $(10,7 \%)$ singletons. Em relação às guildas comportamentais, foram capturadas 14 espécies $(50 \%)$ escavadoras, $12(42,9 \%)$ roladoras e somente duas $(7,1 \%)$ residentes.

Entre os tipos de iscas utilizados, a maior riqueza de espécies (25) foi encontrada nas armadilhas iscadas com excremento humano, enquanto as iscadas com peixe apodrecido apresentaram 21 espécies. $\mathrm{O}$ teste de rarefação demonstrou haver diferença significativa na riqueza de espécies entre os tipos de iscas utilizados para

Tab. II. Proporção observada de indivíduos (\%) nas armadilhas iscadas com peixe apodrecido (PA) e excremento humano (EX), e classificação da guilda trófica (GT) e comportamental (GC) das espécies de Scarabaeinae capturadas em áreas florestais em Silveira Martins, Rio Grande do Sul, Brasil, entre novembro de 2010 e janeiro de $2011(\mathrm{G}$, generalista; * indica preferência alimentar pela coprofagia [C] ou necrofagia [N] [ $\geq 60$ e $<80 \%$ de queda]). Singletons e doubletons são espécies que apresentaram somente um ou dois indivíduos, respectivamente, não sendo classificadas em guildas tróficas.

\begin{tabular}{|c|c|c|c|c|c|c|}
\hline \multirow{2}{*}{ Espécies } & \multicolumn{4}{|c|}{ Iscas } & \multirow{2}{*}{ GT } & \multirow{2}{*}{$\mathrm{GC}$} \\
\hline & PA & $\%$ & EX & $\%$ & & \\
\hline Ateuchus aff. carbonarius & 1 & 100,0 & 0 & 0,0 & Singleton & Escavadora \\
\hline Canthidium aff. dispar & 1 & 20,0 & 4 & 80,0 & Singleton & Escavadora \\
\hline Canthidium aff. trinodosum & 5 & 27,8 & 13 & 72,2 & $\mathrm{G}\left(\mathrm{C}^{*}, \mathrm{~N}\right)$ & Escavadora \\
\hline Canthidium sp. & 10 & 21,3 & 37 & 78,7 & $\mathrm{G}\left(\mathrm{C}^{*}, \mathrm{~N}\right)$ & Escavadora \\
\hline Canthon amabilis & 22 & 44,9 & 27 & 55,1 & Generalista & Roladora \\
\hline Canthon chalybaeus & 117 & 52,2 & 107 & 47,8 & Generalista & Roladora \\
\hline Canthon laminatus & 1 & 25,0 & 3 & 75,0 & $\mathrm{G}\left(\mathrm{C}^{*}, \mathrm{~N}\right)$ & Roladora \\
\hline Canthon latipes & 302 & 37,6 & 502 & 62,4 & $\mathrm{G}\left(\mathrm{C}^{*}, \mathrm{~N}\right)$ & Roladora \\
\hline Canthon lividus & 28 & 63,6 & 16 & 36,4 & $\mathrm{G}\left(\mathrm{C}, \mathrm{N}^{*}\right)$ & Roladora \\
\hline Canthon aff. luctuosus & 29 & 61,7 & 18 & 38,3 & $\mathrm{G}\left(\mathrm{C}, \mathrm{N}^{*}\right)$ & Roladora \\
\hline Canthon oliverioi & 0 & 0,0 & 3 & 100,0 & Coprófaga & Roladora \\
\hline Canthon quinquemaculatus & 2 & 100,0 & 0 & 0,0 & Doubleton & Roladora \\
\hline Coprophanaeus saphirinus & 41 & 65,1 & 22 & 34,9 & $\mathrm{G}\left(\mathrm{C}, \mathrm{N}^{*}\right)$ & Escavadora \\
\hline Deltochilum brasiliense & 16 & 36,4 & 28 & 63,6 & $\mathrm{G}\left(\mathrm{C}^{*}, \mathrm{~N}\right)$ & Roladora \\
\hline Deltochilum morbillosum & 4 & 100,0 & 0 & 0,0 & Necrófaga & Roladora \\
\hline Deltochilum rubripenne & 15 & 60,0 & 10 & 40,0 & $\mathrm{G}\left(\mathrm{C}, \mathrm{N}^{*}\right)$ & Roladora \\
\hline Deltochilum sculpturatum & 46 & 57,5 & 34 & 42,5 & Generalista & Roladora \\
\hline Dichotomius aff. acuticornis & 1 & 50,0 & 1 & 50,0 & Doubleton & Escavadora \\
\hline Dichotomius assifer & 1 & 50,0 & 1 & 50,0 & Doubleton & Escavadora \\
\hline Dichotomius nisus & 0 & 0,0 & 2 & 100,0 & Doubleton & Escavadora \\
\hline Eurysternus caribaeus & 10 & 14,3 & 60 & 85,7 & Coprófaga & Residente \\
\hline Eurysternus parallelus & 2 & 7,1 & 26 & 92,9 & Coprófaga & Residente \\
\hline Ontherus azteca & 0 & 0,0 & 2 & 100,0 & Doubleton & Escavadora \\
\hline Ontherus sulcator & 0 & 0,0 & 2 & 100,0 & Doubleton & Escavadora \\
\hline Onthophagus aff. hirculus & 0 & 0,0 & 2 & 100,0 & Doubleton & Escavadora \\
\hline Onthophagus aff. tristis & 4 & 11,8 & 30 & 88,2 & Coprófaga & Escavadora \\
\hline Phanaeus splendidulus & 0 & 0,0 & 2 & 100,0 & Doubleton & Escavadora \\
\hline Uroxys aff. dilaticollis & 0 & 0,0 & 1 & 100,0 & Singleton & Escavadora \\
\hline Número de indivíduos & 658 & & 953 & & & \\
\hline Número de espécies & 21 & & 25 & & & \\
\hline
\end{tabular}


a captura de Scarabaeinae conforme a não sobreposição dos desvios-padrão calculados para fezes e carne de peixe (peixe em decomposição [ \pm desvio-padrão]: $21 \pm$ 0,00 espécies; excremento humano: $23,5 \pm 1,06$ espécies - Fig. 2). O maior número de indivíduos $(59,2 \%)$ também foi encontrado nas armadilhas iscadas com fezes humanas, porém não houve diferença significativa entre os dois tipos de iscas $(\mathrm{H}=1,614$; g.l. $=1 ; \mathrm{p}=0,20)$.

Conforme o menor valor estimado pelos estimadores de riqueza houve uma captura mínima de $92,8 \%$ da fauna de Scarabaeinae estimada para os fragmentos amostrados (Tab. III). Para as armadilhas iscadas com excremento humano a captura mínima aproximada foi de $89,2 \%$, enquanto que para as iscadas com peixe apodrecido foi de $96,6 \%$.

Tab. III. Riqueza observada e estimada ( \pm desvio-padrão) para os dois tipos de iscas utilizados (peixe apodrecido e excremento humano) para a captura de Scarabaeinae em áreas florestais em Silveira Martins, Rio Grande do Sul, Brasil, entre novembro de 2010 e janeiro de 2011.

\begin{tabular}{lccc}
\hline \multirow{2}{*}{ Estimadores } & \multicolumn{2}{c}{ Iscas } & \\
\cline { 2 - 3 } & $\begin{array}{c}\text { Peixe } \\
\text { apodrecido }\end{array}$ & $\begin{array}{c}\text { Excremento } \\
\text { humano }\end{array}$ & Total \\
\hline Riqueza observada & 21 & 25 & 28 \\
Bootstrap & $20,3 \pm 1,4$ & $23,5 \pm 4,0$ & $27,0 \pm 3,4$ \\
Chao 1 & $20,7 \pm 2,3$ & $22,3 \pm 0,5$ & $26,0 \pm 1,0$ \\
Chao 2 & $20,5 \pm 1,9$ & $26,6 \pm 4,1$ & $29,1 \pm 3,6$ \\
Jackknife 1 & $21,6 \pm 1,4$ & $24,9 \pm 2,6$ & $28,6 \pm 2,6$ \\
Jackknife 2 & $22,1 \pm 3,0$ & $25,8 \pm 7,1$ & $29,4 \pm 6,3$ \\
\hline
\end{tabular}

\section{DISCUSSÃO}

A maioria das espécies capturadas é comum na região central do Rio Grande do Sul. Apenas Canthon laminatus Balthasar (1939) não possuía ainda registros para esta região (P. G. da Silva, dados inéditos), citada apenas para o município de Porto Alegre (Balthasar, 1939). Aparentemente, C. laminatus distribui-se por fragmentos florestais subtropicais na Argentina, Brasil e Paraguai (MARTÍNEZ, 1959).

Conforme os estimadores de riqueza houve êxito na captura da assembleia de Scarabaeinae nos fragmentos amostrados, onde a inclusão de espécies a serem capturadas com um maior número de amostragens é baixa. O número registrado de espécies (28) está próximo ao observado por P. G. da Silva (dados inéditos) no município de Santa Maria, Rio Grande do Sul, que listou 33 espécies utilizando armadilhas de queda iscadas com diferentes recursos. Apenas $C$. laminatus, $O$. aff. hirculus e $U$. aff. dilaticollis não foram registradas no inventário apresentado por P. G. da Silva (dados inéditos). A riqueza de espécies observada em nosso estudo é igual à encontrada por Audino et al. (2011) em bosque de eucalipto do bioma Pampa, município de Bagé, sul do Rio Grande do Sul, onde o número comum de espécies em ambos foi de cinco. Entretanto, a riqueza é bem superior àquela encontrada por SiLva et al. (2008, 2009) e SILVA (2011), onde estes autores capturaram 16,

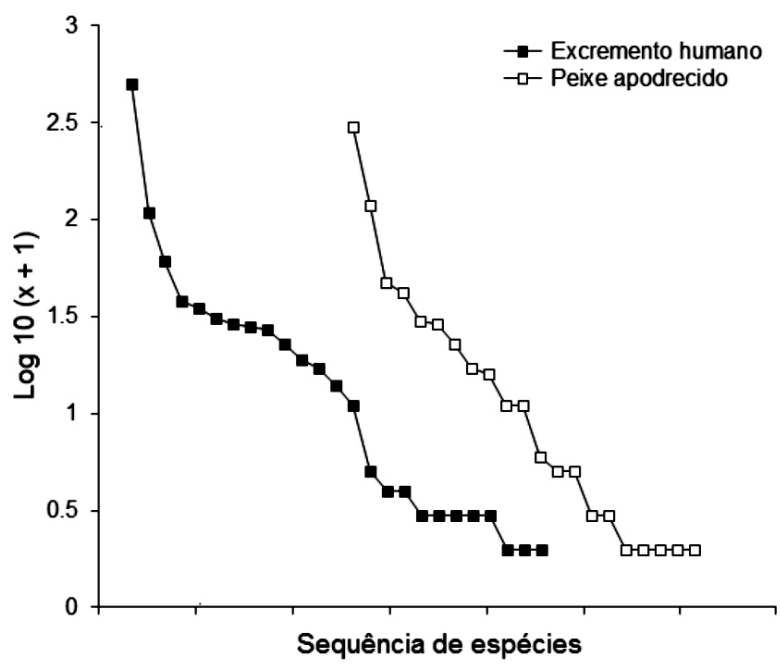

Fig. 1. Distribuição em ranques da abundância $(\mathrm{N})$ das espécies de Scarabaeinae capturadas através de armadilhas de queda iscadas com excremento humano e peixe apodrecido em três áreas florestais em Silveira Martins, Rio Grande do Sul, Brasil, entre novembro de 2010 e janeiro de 2011. A abundância relativa de cada espécie foi transformada em logaritmo de base $10(\mathrm{~N}+1)$.

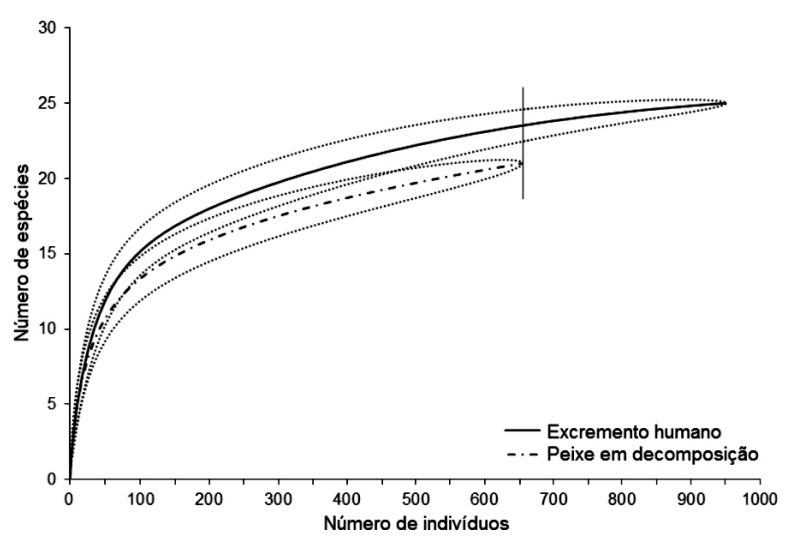

Fig. 2. Teste de rarefação entre as armadilhas iscadas com excremento humano e peixe em decomposição utilizadas para a captura de Scarabaeinae em três áreas florestais em Silveira Martins, Rio Grande do Sul, Brasil, entre novembro de 2010 e janeiro de 2011. Linha vertical indica o ponto de comparação entre os tipos de iscas. Linhas pontilhadas indicam o desvio-padrão.

17 e 13 espécies de Scarabaeinae, respectivamente, em ecótono natural de campo e floresta, uma área de campo nativo, e duas áreas de plantas exóticas (eucalipto e capim annoni), também no município de Bagé.

$\mathrm{O}$ número de singletons (três) e doubletons (oito) neste estudo foi elevado (39,2\% do número de espécies amostradas) e $73 \%$ dos indivíduos capturados pertenceram a somente quatro espécies. Segundo HALFFTER (1991), em florestas neotropicais Scarabaeinae é representada por poucas espécies abundantes e muitas espécies com número reduzido de indivíduos. Este padrão tem sido encontrado frequentemente em estudos realizados tanto em ecossistemas florestais (e.g. LouZAda \& Lopes, 1997; VAZ-De-Mello, 1999; Medri \& LOPES, 2001; MilHomem et al., 2003; ENDRES et al., 2007; LouZADA et al., 2007; Filgueiras et al., 2009; HeRnÁNDEZ 
\& VAZ-De-Mello, 2009; Lopes et al., 2011) quanto campestres (e.g. Matavelli \& LouZADA, 2008; Koller et al., 2007; LouZADA \& SiLVA, 2009; LouZADA et al., 2010; Lopes et al., 2011) no Brasil e em vários outros países neotropicais (e.g. HALFFTER et al., 1992; Morelli et al., 2002; Spector \& Ayzama, 2003; Martínez et al., 2009). Porém, espécies que estiveram representadas por poucos indivíduos, como C. quinquemaculatus, Dichotomius assifer (Eschscholtz, 1822), D. nisus, O. sulcator e $P$. splendidulus, foram abundantes em outros estudos e regiões do Brasil (e.g. LouZADA \& Lopes, 1997; LouZADA et al., 2007; HeRnÁNDEZ \& VAZ-DE-Mello, 2009; Audino et al., 2011; Lopes et al., 2011).

Embora as amostragens tenham ocorrido no período de maior abundância de Scarabaeinae (HALFFTER \& Matthews, 1966), é possível que o estudo não tenha sido suficientemente amplo para abranger o período de maior abundância destas espécies ou pode ter havido viés no protocolo de amostragem que resultou em uma sub-representação da abundância de algumas espécies (Navarrete, 1996). Entretanto, P. G. da Silva (dados inéditos) encontrou os maiores números destas mesmas espécies durante o período mais quente do ano (primavera-verão). Dessa forma, é possível que estas espécies tenham ocorrido em baixa abundância devido a características estruturais do habitat, da paisagem, ou de disponibilidade de alimento específico, o que possibilitou a maior abundância de outras espécies.

O maior número de espécies de hábito alimentar generalista em relação às especialistas (coprófagas ou necrófagas) é um padrão comumente encontrado nas assembleias neotropicais de Scarabaeinae (HALfFTER \& Matthews, 1966; Halffter, 1991), embora a alta competição em recursos escassos e efêmeros como excrementos e carcaças possa levar a uma alta especialização trófica (PeCK \& Forsyth, 1982; HANSKi \& CAMBEForT, 1991). A generalidade trófica tende a diminuir a competição por alimentos escassos e efêmeros, permitindo às espécies de Scarabaeinae uma melhor utilização dos recursos do ecossistema, enquanto a especificidade parece restringir a ocupação de novos habitats onde seu alimento não está disponível (SiLva, 2011). A capacidade dos escarabeíneos utilizarem alimentos alternativos teria contribuído para a alta diversidade de espécies deste grupo na região Neotropical (Halffter \& HalfFter, 2009).

Canthon oliverioi, E. caribaeus, E. parallelus Castelnau, 1840 e Onthophagus aff. tristis Harold, 1873, classificadas como coprófagas, são muito atraídas por excremento humano fresco (Pereira \& Martínez, 1956; MarTíneZ, 1959; GÉnIER, 2009), mas podem ser atraídas por armadilhas iscadas com carne e/ou banana em decomposição (P. G. da Silva, dados inéditos). A única espécie classificada como necrófaga, D. morbillosum, pode também ser capturada em armadilhas iscadas com excremento humano (P. G. da Silva, dados inéditos).

Em relação às espécies classificadas como generalistas, Canthidium aff. trinodosum (Boheman, 1858), Canthidium sp., C. laminatus, C. latipes e Deltochilum brasiliense (Castelnau, 1840) tiveram preferência pela coprofagia. P. G. da Silva (dados inéditos) encontrou maior número de indivíduos de $C$. aff. trinodosum, Canthidium sp., C. latipes e D. brasiliense em armadilhas iscadas com excremento humano, mas todas foram também atraídas por carne e banana em decomposição. Canthon latipes possui registros de captura em cortes de liana (cipó), provavelmente atraída pelo odor forte desprendida da mesma (Pereira \& MARTíneZ, 1956), além de ser frequente em excrementos de grandes herbívoros (MARTínez, 1959). Segundo Martínez (1959), C. laminatus é encontrada em excrementos humanos.

As espécies Canthon lividus Blanchard, 1845, Canthon aff. luctuosus Harold, 1868, C. saphirinus e Deltochilum rubripenne (Gory, 1831) não foram classificadas nas categorias anteriores, mas apresentaram preferência pela necrofagia conforme o critério utilizado neste estudo. P. G. da Silva (dados inéditos) coletou-as também em armadilhas iscadas com banana apodrecida. Martínez (1959) cita C. saphirinus e D. rubripenne como coprófagas encontradas em excrementos humanos e de herbívoros.

Canthon amabilis Balthasar, 1939, C. chalybaeus e D. sculpturatum foram agrupadas apenas como generalistas, sem indicação de preferência alimentar, pois tiveram proporções de indivíduos semelhantes entre os dois tipos de iscas utilizados. P. G. da Silva (dados inéditos) registrou maior número de indivíduos destas espécies em armadilhas iscadas com carne apodrecida. MarTínez (1959) citou D. sculpturatum como necrófaga, e C. chalybaeus como generalista, encontrada em carcaças e excrementos. Dessa forma, apesar da utilização de critérios para a classificação das espécies de escarabeíneos os quais sugerem que as mesmas são especialistas, a utilização de outros recursos em sua alimentação pode sugerir que as espécies classificadas como de hábito alimentar especialistas possam ser generalistas (AlmeidA \& LouZADA, 2009) quando o alimento preferencial não está disponível, confirmando o padrão mencionado.

Houve um maior número de espécies escavadoras em relação aos demais grupos comportamentais de alocação do recurso alimentar, sendo este um padrão comum em florestas neotropicais (HALFFTER et al., 1992; LOUZADA \& LOPES, 1997). Este padrão de distribuição das espécies pelas guildas comportamentais parece ser fruto da diversidade local das tribos de Scarabaeinae em florestas na região Neotropical (LouZADA \& Lopes, 1997). Por outro lado, a proporção de tribos, gêneros e espécies capturada com armadilhas iscadas restringe-se apenas à maior parte da fauna de Scarabaeinae, uma vez que vários outros grupos de espécies geralmente não são atraídos por este método de amostragem (e.g. VAZDe-Mello et al., 1998; VaZ-DE-Mello, 2007; Larsen 
et al., 2009; VAZ-De-Mello \& GÉNier, 2009). Dessa forma, a interação entre a diversidade dos grupos no neotrópico e a utilização de iscas compatíveis com o hábito alimentar da maioria das espécies parece refletir o padrão encontrado entre diferentes estudos que utilizam iscas para a captura de escarabeíneos.

As armadilhas iscadas com excremento humano apresentaram a maior riqueza de espécies e de espécies exclusivas (sete), quando comparadas às iscadas com peixe apodrecido (três espécies exclusivas). Excremento humano é uma das mais atrativas iscas para a fauna neotropical de Scarabaeinae (HALFFTER \& Matthews, 1966; FAlqueto et al., 2005; LARSen et al., 2006; Filgueiras et al., 2009), uma vez que a grande maioria dos escarabeíneos consome principalmente fezes de grandes mamíferos, fruto de processos evolutivos (Halffter \& Matthews, 1966; Hanski \& Cambefort, 1991; Davis et al., 2002; Simmons \& RidsDill-Smith, 2011). Em segundo plano, a fauna de Scarabaeinae se alimenta de restos de animais mortos (Halffter \& Matthews, 1966), processo particularmente importante na região Neotropical devido à escassez de grandes mamíferos presente nas florestas, o que resulta em menor oferta de excrementos (HALFFTER, 1991). HALFFTER \& EDMONDS (1982) sugerem que os escarabeíneos podem apresentar necessidades fisiológicas sazonais em função de seu estágio reprodutivo, o que pode também explicar a utilização de distintos recursos alimentares em diferentes momentos de seu ciclo de vida.

Assembleias neotropicais de Scarabaeinae de regiões com clima subtropical apresentam menor número de espécies do que comunidades tropicais (HalfFter \& Matthews, 1966). Porém, a competição por recursos escassos e efêmeros como carcaças e excrementos parece manter-se inalterada independentemente do clima do ecossistema. Nas regiões subtropicais, onde a oferta de alimento parece ser menor e condicionada por fatores climáticos, hábitos alimentares generalistas presente nos escarabeíneos parece ser um mecanismo adaptativo importante para a manutenção das espécies, especialmente quando o alimento preferencial esteja temporal ou espacialmente limitado (HowdEN \& Young, 1981; LOUZADA \& LoPES, 1997).

\section{CONCLUSÃO}

A fauna de Scarabaeinae capturada nos fragmentos de Mata Atlântica no sul do Brasil apresentou elevada riqueza, e a maioria das espécies e dos indivíduos capturados foi atraída para as armadilhas iscadas com fezes humanas. A maior parte dos escarabeíneos foi representada por espécies de hábito alimentar generalista e comportamento escavador. Dessa forma, a comunidade de escarabeíneos deste estudo segue os padrões gerais de estrutura trófica e comportamental de Scarabaeinae encontrados por toda a região Neotropical.
Agradecimentos. Os autores agradecem aos proprietários pela disponibilização e autorização para a realização das coletas em fragmentos dentro de suas propriedades. Agradecimentos ao Dr. Fernando Vaz-de-Mello pelo auxílio na identificação dos espécimes; à Coordenação de Aperfeiçoamento de Pessoal de Nível Superior (CAPES) pela concessão de bolsa de estudos ao primeiro autor e aos revisores pelas valiosas contribuições.

\section{REFERÊNCIAS BIBLIOGRÁFICAS}

Almeida, S. S. P. \& Louzada, J. N. C. 2009. Estrutura da comunidade de Scarabaeinae (Scarabaeidae: Coleoptera) em fitofisionomias do Cerrado e sua importância para a conservação. Neotropical Entomology 38:32-43

Audino, L. D.; Silva, P. G.; Nogueira, J. M.; Moraes, L. P. \& Vaz-DeMello, F. Z. 2011. Scarabaeinae (Coleoptera, Scarabaeidae) de um bosque de eucalipto introduzido em uma região originalmente campestre. Iheringia, Série Zoologia 101(1-2):121-126.

Ayres, M.; Ayres-Jr., M.; Ayres, D. L. \& Santos, A. A. S. 2007. BioEstat 5.0. Aplicações estatísticas nas áreas das ciências biomédicas. Belém, Universidade Federal do Pará. 364p.

Balthasar, V. 1939. Eine Vorstudie zur Monographie der Gattung Canthon Hffsg. (10. Beitrag zur Kenntnis der Scarababaeiden der neotropischen Region). Folia Zoologica et Hydrobiologica 9(2):179-238.

Bencke, G. A. 2009. Diversidade e conservação da fauna dos campos do sul do Brasil. In: Pillar, V. P.; Müller, S. C.; Castilhos, Z. M. S. \& JaCQues, A. V. A. eds. Campos Sulinos - conservação e uso sustentável da biodiversidade. Brasília, Ministério do Meio Ambiente. p.101-121.

CAmbefort, Y. \& Hanski, I. 1991. Dung beetle population biology. In Hanski, I. \& CAmbefort, Y. eds. Dung beetle ecology. Princeton, Princeton University Press. p.36-50.

Colwell, R. K. 2004. EstimateS: Statistic estimation of species richness and shared species from samples. Version 7.0 Disponível em: <http://viceroy.eeb.uconn.edu/EstimateS $>$. Acesso em: 15.11.2004.

Colwell, R. K. \& Coddington, J. A. 1994. Estimating terrestrial biodiversity through extrapolation. Philosophical Transactions of the Royal Society B 345:101-118.

Davis, A. L. V.; Scholtz, C. H. \& Philips, T. K. 2002. Historical biogeography of scarabaeine dung beetles. Journal of Biogeography 29:1217-1256.

Davis, A. L.; Scholtz, C. H.; Dooley, P.; Bham, N. \& Kryger, U. 2004. Scarabaeine dung beetles as indicators of biodiversity, habitat transformation and pest control chemicals in agro-ecosystems. South African Journal of Science 100:415-424.

Doube, B. M. 1991. Dung beetle of Southern Africa. In: HansKi, I. \& CAmbefort, Y. eds. Dung beetle ecology. Princeton, Princeton University Press. p.133-155.

Endres, A. A.; Creão-Dunarte, A. J. \& Hernández, M. I. M. 2007. Diversidade de Scarabaeidae s. str. (Coleoptera) da Reserva Biológica Guaribas, Mamanguape, Paraíba, Brasil: uma comparação entre Mata Atlântica e Tabuleiro Nordestino. Revista Brasileira de Entomologia 51(1):67-71.

Escobar, F.; Halffter, G. \& Arellano, L. 2007. From forest to pasture: an evaluation of the influence of environment and biogeography on the structure of dung beetle (Scarabaeinae) assemblages along three altitudinal gradients in the Neotropical region. Ecography 30:193-208.

Falqueto, S. A.; Vaz-de-Mello, F. Z. \& Schoereder, J. H. 2005. Are fungivorous Scarabaeidae less specialist? Ecología Austral 15:17-22.

FEINSINGER, P. 2001. Designing field studies for biodiversity conservation. Washington, Island Press. 236p.

Filgueiras, B. K. C.; Iannuzzi, L. \& Leal, I. R. 2011. Habitat fragmentation alters the structure of dung beetles communities in the Atlantic Forest. Biological Conservation 144:362-369.

Filgueiras, B. K. C.; Liberal, C. N.; Aguiar, C. D. M. Hernández, M. I. M. \& IANNUZZI, L. 2009. Attractivity of omnivore, carnivore and herbivore mammalian dung to Scarabaeinae (Coleoptera Scarabaeidae) in a tropical Atlantic Forest remnant. Revista Brasileira de Entomologia 53(3):422-427.

Gardner, T. A.; Hernández, M. I. M.; Barlow, B. \& Peres, C. A. 2008 Understanding the biodiversity consequences of habitat change: the value of secondary and plantation forests for Neotropical dung beetles. Journal of Applied Ecology 45:883-893. 
GÉNIER, F. 2009. Le genre Eurysternus Dalman, 1824 (Scarabaeidae: Scarabaeinae: Oniticellini), revision taxonomique et clés de determination illustrées. Sofia, Pensoft. 430p.

Gill, B. D. 1991. Dung beetles in tropical American forests. In: Hanski, I. \& CAmbefort, Y. eds. Dung beetle ecology. Princeton, Princeton University Press. p.211-229.

Halffter, G. 1991. Historical and ecological factors determining the geographical distribution of beetles (Coleoptera: Scarabaeidae: Scarabaeinae). Folia Entomologica Mexicana 82:195-238.

Halffter, G. \& Arellano, L. 2002. Response of dung beetles diversity to human-induced changes in a tropical landscape. Biotropica 34(1): 144-154

HalfFter, G. \& Edmonds, W. D. 1982. The nesting behavior of dung beetles (Scarabaeinae): An ecologic and evolutive approach México D. F., Man and Biosphere Program UNESCO. 177p.

Halffter, G. \& Favila, M. E. 1993. The Scarabaeidae (Insecta: Coleoptera) an animal group for analyzing, inventorying and monitoring biodiversity in tropical rainforest and modified landscapes. Biology International 27:15-21.

Halffter, G. \& Halffter, V. 2009. Why and where coprophagous beetles (Coleoptera: Scarabaeinae) eat seeds, fruits or vegetable detritus. Boletín de la Sociedad Entomologica Aragonesa 45:1 22.

Halffter, G. \& Matthews, E. G. 1966. The natural history of dung beetles of the subfamily Scarabaeinae (Coleoptera: Scarabaeidae). Folia Entomologica Mexicana 12/14:1-312.

Halffter, G.; Favila, M. E. \& Halffter, V. 1992. A comparative study of the structure of the scarab guild in Mexican tropical rain forests and derived ecosystems. Folia Entomologica Mexicana 84:131156.

Hammer, O.; Harper, D. A. T. \& Ryan, P. D. 2001. PAST: Palaeontological Statistics software package for education and data analysis. Palaeontologia Electronica 4(1):9.

Hanski, I. \& Cambefort, Y. 1991. Competition in dung beetles. In: Hanski, I. \& Camberort, Y. eds. Dung beetle ecology. Princeton, Princeton University Press. p. 305-329.

Hernández, M. I. M. \& VAZ-DE-Mello, F. Z. 2009. Seasonal and spatial species richness varation of dung beetle (Coleoptera, Scarabaeidae s. str.) in the Atlantic Forest of southeastern Brazil. Revista Brasileira de Entomologia 53(4):607-613.

Howden, H. F. \& Young, O. P. 1981. Panamanian Scarabaeinae: Taxonomy, distribution, and habits (Coleoptera, Scarabaeidae) Contribution of the American Entomological Institute 18:1204.

Hulbert, S. H. 1971. Nonconcept of species diversity: A critique and alternative parameters. Ecology 52(4):577-586.

IBGE, Instituto Brasileiro de Geografia e Estatística. 2011. Cidades - RS. Disponível em: <http://www.ibge.gov.br/cidadesat/painel/ painel.php?codmun $=432065>$. Acesso em: 10.04.2011.

KLEIN, B. C. 1989. Effects of forest fragmentation on dung and carrion beetle communities in central Amazonia. Ecology 70(6):1715 1725.

Koller, W. W.; Gomes, A.; Rodrigues, S. R. \& Goiozo, P. F. I. 2007. Scarabaeidae e Aphodiidae coprófagos em pastagens cultivadas em área do cerrado sul-mato-grossense. Revista Brasileira de Zoociências 9(1):81-93.

Larsen, T. H. \& Forsyth, A. 2005. Trap spacing and transect design for dung beetle biodiversity studies. Biotropica 37(2):322-325.

Larsen, T. H.; Lopera, A. \& Forsyth, A. 2006. Extreme trophic and habitat specialization by Peruvian dung beetles (Coleoptera: Scarabaeidae: Scarabaeinae). The Coleopterists Bulletin 60:315324.

Larsen, T. H.; Lopera, A.; Forsyth, A. \& Génier, F. 2009. From coprophagy to predation: a dung beetle that kills millipedes. Biology Letters 5:152-155.

Lobo, J. M.; Martín-Piera, F. \& Veiga, C. M. 1988. Las trampas pitfall con sebo, sus posibilidades en el estudio de las comunidades coprófagas de Scarabaeoidea (Col.). I. Características determinantes de su capacidad de captura. Revue d'Ecologie et de Biologie du Sol 25:77-100.

Lopes, J.; Korasaki, V.; Catelli, L. L.; Marçal, V. V. M. \& Nunes, M. P. B. P. 2011. A comparison of dung beetle assemblage structure (Coleoptera: Scarabaeidae: Scarabaeinae) between an Atlantic forest fragment and adjacent abandoned pasture in Paraná, Brazil. Zoologia 28(1):72-79.
LouZADA, J. N. C. \& Lopes, F. S. 1997. A comunidade de Scarabaeidae copro-necrófagos (Coleoptera) de um fragmento de Mata Atlântica. Revista Brasileira de Entomologia 41(1):117-121.

Louzada, J. N. C. \& Silva, P. R. C. 2009. Utilisation of introduced Brazilian pastures ecosystems by native dung beetles: diversity patterns and resource use. Insect Conservation and Diversity 2:45-52.

Louzada, J. N. C.; Lopes, F. S. \& VaZ-De-Mello, F. Z. 2007. Structure and composition of a dung beetle community (Coleoptera, Scarabaeinae) in a small forest patch from Brazilian Pantanal. Revista Brasileira de Zoociências 9(2):199-203.

Louzada, J.; Lima, A. P.; Matavelli, R.; Zambaldi, L. \& Barlow, J. 2010. Community structure of dung beetles in Amazonian savannas: role off ire disturbance, vegetation and landscape structure. Landscape Ecology 25:631-641.

Martínez, A. 1959. Catalogo de los Scarabaeidae Argentinos (Coleoptera). Revista del Museo Argentino de Ciencias Naturales "Bernardino Rivadavia" 5:1-126.

Martínez, N. J.; García, H.; Pulido, L. A.; Ospino, D. \& Narváez, J. C. 2009. Escarabajos coprófagos (Coleoptera: Scarabaeinae) de la Vertiente Noroccidental, Sierra Nevada de Santa Marta, Colombia. Neotropical Entomology 38(6):708-715.

Matavelli, R. A. \& LouzadA, J. N. C. 2008. Invasão de áreas de savana intra-amazônicas por Digitonthophagus gazella (Fabricius, 1787) (Insecta: Coleoptera: Scarabaeidae). Acta Amazonica 38(1):153158.

MedRI, Í. M. \& Lopes, J. 2001. Scarabaeidae (Coleoptera) do Parque Estadual Mata dos Godoy e de área de pastagem, no norte do Paraná, Brasil. Revista Brasileira de Zoologia 18(Supl.1):135141.

Milhomem, M. S.; VaZ-De-Mello, F. Z. \& Diniz, I. R. 2003. Técnicas de coleta de besouros copronecrófagos no Cerrado. Revista Agropecuária Brasileira 38(11):1249-1256.

Morelli, E.; GonzÁlez-Vainer, P. \& Baz, A. 2002. Coprophagous beetles (Coleoptera: Scarabaeoidea) in Uruguayan Prairies: abundance, diversity and seasonal occurrence. Studies on Neotropical Fauna and Environment 37:53-57.

Moura, L. DE A. 2003. Coleópteros. In: Fontana, C. S.; Bencke, G. A. \& Reis, R. E. eds. Livro Vermelho da Fauna Ameaçada de no Rio Grande do Sul. Porto Alegre, Edipucrs. p.85-94.

NavarRete, H. J. L. 1996. Is the apparent rarity of Liatongus monstrosus (Bates) (Coleoptera: Scarabaeidae) real or an artifact of collecting? The Coleopterists Bulletin 50:216-220.

Neves, F. S.; Oliveira, V. H. F.; Espírito-Santo, M. M.; Vaz-deMello, F. Z.; Louzada, J.; Sanchez-Azofeifa, A. \& Fernandes, G. W. 2010. Successional and seasonal changes in a community of dung beetles (Coleoptera: Scarabaeinae) in a Brazilian Tropical Dry Forest. Natureza \& Conservação 8(2): 160-164.

Nichols, E.; Spector, S.; Louzada, J.; Larsen, T.; Amezquita, S. \& FAVILA, M. E. 2008. Ecological functions and ecosystem services provided by Scarabaeinae dung beetles. Biological Conservation 141:1461-1474.

Nichols, E.; Larsen, T.; Spector, S.; Davis, A. L.; Escobar, F.; Favila, M. \& Vulinec, K. 2007. Global dung beetle response to tropical forest modification and fragmentation: A quantitative literature review and meta-analyses. Biological Conservation 137:1-19

Peck, S. B. \& Forsyth, A. 1982. Composition, structure, and competitive behaviour in a guild of Ecuadorian rain forest dung beetles (Coleoptera; Scarabaeidae). Canadian Journal of Zoology 60:1624-1634.

Pell, M. C.; FinLAYSON, B. L. \& MCMAHON, T. A. 2007. Updated world map of the Köppen-Geiger climate classification. Hydrology and Earth System Sciences 11:1633-1644.

Pereira, F. S. \& Martínez, A. 1956. Os gêneros de Canthonini americanos (Col. Scarabaeidae). Revista Brasileira de Entomologia 6:91-192.

Schoolmeesters, P.; Davis, A. L. V.; Edmonds, W. D.; Gill, B.; Mann, D.; Moretto, P.; Price, D.; Reid, C.; Spector, S. \& VAZ-DeMello, F. Z. 2010. ScarabNet Global Taxon Database (version 1.5). Disponível em: <http://216.73.243.70/scarabnet/results $\mathrm{htm}>$. Acesso em: 16.08.2010.

Silva, P. G. 2011. Dung beetles (Coleoptera: Scarabaeidae: Scarabaeinae) of two non-native habitats in Bagé, Rio Grande do Sul, Brazil. Zoological Studies 50(5):546-559. 
Silva, P. G.; Garcia, M. A. R. \& Vidal, M. B. 2008. Besouros copronecrófagos (Coleoptera: Scarabaeidae stricto sensu) coletados em ecótono natural de campo e mata em Bagé, RS. Ciência e Natura 30(2):71-91.

. 2009. Besouros copro-necrófagos (Coleoptera: Scarabaeidae sensu stricto) do município de Bagé, RS (Bioma Campos Sulinos). Biociências 17(1):33-43.

Simmons, L. W. \& Ridsdill-Smith, T. J. 2011. Reproductive competition and its impact on the evolution and ecology of dung beetles. In: Simmons, L. W. \& RidsDill-Smith, T. J. eds. Ecology and evolution of dung beetles. Oxford, Blackwell Publishing. p. 1-20.

Spector, S. \& Ayzama, S. 2003. Rapid turnover and edge effects in dung beetle assemblages (Scarabaeidae) at a Bolivian Neotropical forest-savanna ecotone. Biotropica 35:394-404.

VAZ-de-Mello, F. Z. 1999. Scarabaeidae s. str. (Coleoptera: Scarabaeoidea) de um fragmento de Floresta Amazônica no estado do Acre, Brasil. I. Taxocenose. Anais da Sociedade Entomológica do Brasil 28(3):447-453.

2000. Estado de conhecimento dos Scarabaeidae s. str. (Coleoptera: Scarabaeoidea) do Brasil. In: Martín-Piera, F.; Morrone, J. J. \& Melic, A. eds. Hacia un proyecto CYTED para el inventario y estimación de la diversidad entomológica en Iberoamérica. Zaragoza, Sociedad Entomologica Aragonesa p.181-195.

2007. Revision and phylogeny of the dung beetle genus Zonocopris Arrow 1932 (Coleoptera: Scarabaeidae: Scarabaeinae), a phoretic of land snails. Annales de la Société Entomologique de France 43(2):231-239.

VAZ-DE-Mello, F. Z. \& GÉnier, F. 2009. Notes on the behavior of Dendropaemon Perty and Tetramereia Klages (Scarabaeidae: Scarabaeinae: Phanaeini). The Coleopterists Bulletin 63(3):364366.

VaZ-De-Mello, F. Z.; LouZada, J. N. C. \& Schoereder, J. H. 1998. New data and comments on Scarabaeidae (Coleoptera: Scarabaeoidea) associated with Attini (Hymenoptera: Formicidae). The Coleopterists Bulletin 52(3):209-216.

Vaz-de-Mello, F. Z.; Edmonds, W. D.; Ocampo, F. \& Schoolmeesters, P. 2011. A multilingual key to the genera and subgenera of the subfamily Scarabaeinae of the New World (Coleoptera: Scarabaeidae). Zootaxa 2854:1-73.

Verdú, J. R.; Moreno, C. E.; Sánchez-Rojas, G.; Numa, C.; Galante, E. \& HalfFter, G. 2007. Grazing promotes dung beetle diversity in the xeric landscape of a Mexican Biosphere Reserve. Biological Conservation 140:308-317. 